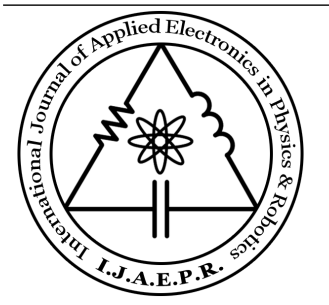

Research Article

\title{
Modified Pole-Placement Controller via Takagi-Sugeno Fuzzy Modelling Approach: Applied to a Load Frequency Stability Problem
}

\author{
Rabiu Sabiu Shehu ${ }^{1, *}$, Hamisu Usman ${ }^{1}$, Ado Dan-Isa ${ }^{2}$ \\ (1) Department of Electrical and Electronics Engineering, Kaduna Polytechnic, Kaduna, Nigeria, P.M.B. 2021, kaduna, Nigeria. \\ (2) Department of Electrical Engineering, Faculty of Technology, Bayero University, Kano, P.M.B. 2011, Kano, Nigeria.
}

\section{Article history:}

Received 08 January 2014 Reviewed 18 January 2014 Revised 24 January 2014 Accepted 26 January 2014

Published 28 January 2014
Abstract. The paper aims at incorporating intelligent modelling paradigm-the Takagi-Sugeno(TS) model and a conventional pole placement control methods in achieving stability for a single area power system network. Four different operating points describing four different local linear state models are used in obtaining the TS fuzzy model, state estimation based on Ackermann's principle was used to determine the state feedback matrix for the four selected operating points, the system in open loop and in closed loop is simulated at varying parameter conditions. Results indicating effectiveness of the developed controller over other reported control method are generated.

Keywords: T-S Fuzzy Model, Stability, State Feedback Gain Matrix, State Estimation, Parameter.

\section{Introduction}

Fuzzy logic control is a soft computing control method. It employs a number of statements or rules each expressed in IF-THEN format as a basis for implementing a control law. The rules are representation of knowledge collected from an expert concerning step by step control of a given physical system. Two fuzzy modelling approaches are Mamdani and Takagi-Sugeno (T-S) models. While in the Mamdani modelling fuzzy functions are use in the premise (input) and consequence (output) parts of a rule, the T-S fuzzy model has static or dynamic model in the consequence part. Many formulations of the Mamdani fuzzy models used in the context of control are reported in references ${ }^{[1-3]}$. The T-S fuzzy model has rule that has static or dynamic equation in the rules' output. The T-S fuzzy model based method has some merits in that wide range of complex non-linear systems can be represented as a set of local linear models in the consequence part of the rule. Numerous works on control and signal processing have used various forms of T-S fuzzy model ${ }^{[4-6]}$. Specifically for power system control we found some successful works reported in references ${ }^{[4,7,8]}$.

In conventional pole placement control, a desire performance is set by placing the system poles at a desired locations in s-plane. Application of pole placement strategy has also been reported within the frame work of T-S fuzzy model control. Some works can be seen in references $^{[9,10]}$.

\footnotetext{
*Corresponding author: R. S. Shehu ㅁ: +2347037079913

凶: rabiushehu69@gmail.com
}

Due to competitive market demand on the control of industrial systems, the control problem become too complex to handle, partly due to varying nature of the system parameter conditions. Classical control method such as pole placement technique suffers because of lack of adequate math model necessary for developing effective controllers. There is a need therefore to introduce a knowledge base system that could reduce the need for explicit math model. Therefore, we seek to introduce a T-S fuzzy modeling frame work which carries with it, some form of system knowledge into the classical pole placement control design, with the aim of achieving robust control system performance even in the presence of varying system parameter conditions.

In this paper, we proposed a method of modifying a pole placement based control law using a T-S fuzzy model parameters. We shall present results that would shows the effectiveness of the control scheme.

\section{Mathematical Preliminaries}

Consider a general non-linear system given as

$$
\dot{x}(t)=f(x(t))+g(x(t)) u(t)+w(t)
$$

where $x(t) \in \mathbb{R}^{n}$ is state vector, $u(t) \in \mathbb{R}^{m}$ is input control signal, $w(t) \in \mathbb{R}^{p}$ is external disturbance signal, $f$ and $g$ are non-linear smooth functions. Detail discussion on the T-S fuzzy modelling approaches are provided in reference $^{[11]}$. Basically T-S fuzzy modelling system provides a mapping space consisting of a vector $\mathbb{R}^{n} \times \mathbb{R}^{m} \times \mathbb{R}^{p}$ such 
that

$$
\sup _{x(t), u(t) \subset \Phi}\|f(x(t), u(t), w(t))-\Phi(x(t), u(t), w(t))\| \leq \epsilon
$$

where $\epsilon$ is an arbitrary small positive number. To briefly explain the T-S fuzzy concept, let

$$
\Sigma_{i}=\left(A_{i}, B_{i}, D_{i}\right)
$$

be linear space, where $A_{i}, B_{i}$ and $D_{i}$ are parametric matrices of the $i^{t h}$ local linear model, with $i=1,2, \cdots, q$ being an index of triplets, then a T-S fuzzy system mapping function at an instant of time would yield the following.

$$
\phi(x(t), u(t), w(t))=A(\mu) x(t)+B(\mu) u(t)+D(\mu) w(t)
$$

The argument $\mu_{i}$ in the right side of (4) is fuzzy function. A local linear model as output in response to inputs in a local $i^{t h}$ sector of a domain is represented using a rule of the form Rule $i\left(R_{i}\right)$.

if $\left(z_{1}(t)\right.$ is $M_{1}^{i}$ \&\& $z_{2}(t)$ is $M_{2}^{i} \& \& \cdots z_{s}(t)$ is $\left.M_{s}^{i}\right)$

then $\quad \dot{x}(t)=A_{i} x(t)+B_{i} u(t)+D_{i} w(t)$

where $R_{i}$ denotes the $i^{t h}$ fuzzy rule, where $z(t)=$ $\left[z_{1}(t), z_{2}(t), \cdots, z_{s}(t)\right]$ is premise input variable, $M_{j}^{i}=$ $\left[M_{1}^{i}, M_{2}^{i}, \cdots, M_{s}^{i}\right]$ is fuzzy linguistic value quantifying membership degree of an input. Many types of function shapes are used, popular one is the triangular shape shown in Fig. 1.

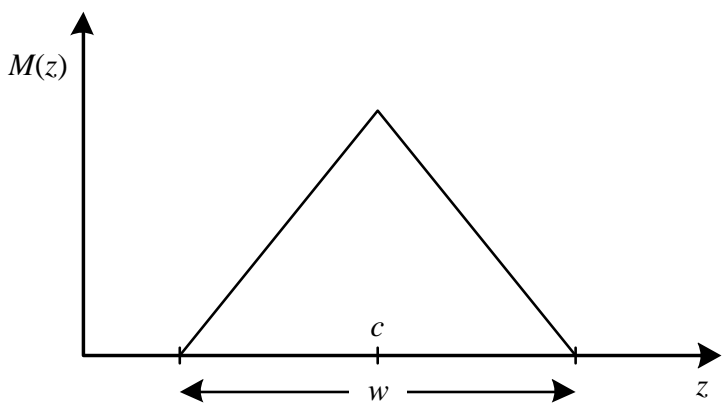

Fig. 1. A triangular fuzzy membership function

Mathematically the shape is expressed as

$$
M\left(z_{i}\right)=\max \left(0,1+\frac{\left|z_{j}-c_{j}\right|}{0.5 \beta_{j}}\right), \quad j=1,2, \cdots, q
$$

where $c_{j}, \beta_{j}$ are centre and width of the triangular shape. The overall T-S fuzzy model resulting from $R_{i}$ after combination and aggregation can be written as

$$
\dot{x}(t)=\sum_{i=1}^{R} \mu_{i}(z(t))\left(A_{i} x(t)+B_{i} u(t)+D_{i} w(t)\right)
$$

where

$$
\mu_{i}(z(t))=\frac{\varsigma_{i}(z(t))}{\sum_{i=1}^{R} \varsigma_{i}(z(t))}
$$

and

$$
\varsigma_{i}(z(t))=\prod_{j}^{s} M_{j}^{i}(z(t))
$$

\subsection{State Feedback Control law and Fuzzy Closed Loop System}

In order to evolve the fuzzy based pole-placement controller, we recalled the concept the Parallel Distributed
Compensation (PDC) used in T-S fuzzy based control ${ }^{[12]}$. In PDC a $j^{t h}$ control law written as

$$
u_{j}=-\mu_{j}(z(t)) K_{j} x(t), \quad j=1,2, \cdots, R
$$

is design to compensate an $i^{\text {th }} \mathrm{T}-\mathrm{S}$ fuzzy rule of $R_{i}$. Where $K_{j}$ is state feedback gain, $\mu_{j}$ is $j^{\text {th }}$ fuzzy scaling factor as defined in (6) and $R$ is number T-S fuzzy rules. If the linear quadratic regulator (LQR) or pole placement design can be use to determine $K_{j}$ the system in (6) with (9) involved is written in a closed loop system as (10).

$$
\dot{x}(t)=\sum_{i=1}^{R} \sum_{j=1}^{R} \mu_{i} \mu_{j}\left(A_{i}-B_{i} K_{i}\right) x(t)
$$

In this paper, we shall determine the gain in (10) using fuzzy pole placement in the context of state estimation technique.

\subsection{Feedback Gain Design Through Pole- Placement}

Let the $i^{\text {th }}$ local linear model in $R_{i}$ behave as define by the characteristic equation

$$
\begin{aligned}
q(s) & =s^{2}+5 s+6 \\
& =0
\end{aligned}
$$

Based on Ackermann's idea ${ }^{[13]}$, we write the gain as

$$
K_{j}=q_{j} \alpha\left(A_{j}\right)
$$

Where $q_{i}$ is $j^{\text {th }}$ last row of the $j^{\text {th }}$ controllability matrix and

$$
\alpha\left(A_{j}\right)=A_{j}^{n}+a_{n-1} A_{j}^{n-1}+\cdots+a_{0} I_{2} .
$$

Having specified the desired dynamics in (11), we could use MATLAB command to generate the appropriate state feedback gain for system in (10).

\section{Application}

To test the effectiveness of the control scheme, we consider the problem of maintaining frequency stability in power system network model shown in Fig. 2.

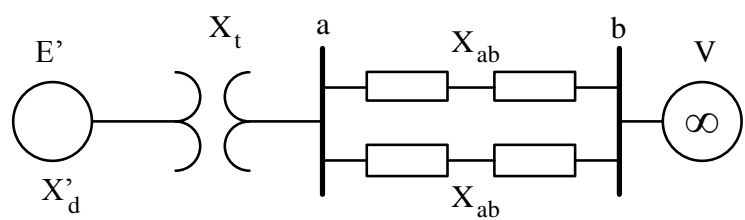

Fig. 2. Single area power system network

Describing the system in state space form

$$
\begin{aligned}
\dot{x} & =A x+B u \\
y & =C x
\end{aligned}
$$

where $A$ is $n \times n$ system matrix, $B$ is $n \times p$ input matrix, $C$ is $m \times n$ output matrix and $y$ is system output. We recalled the approximate parametric linear model of the network reported in reference ${ }^{[14]}$ as

$$
\begin{aligned}
{\left[\begin{array}{c}
\dot{x}_{1} \\
\dot{x}_{2}
\end{array}\right] } & =\left[\begin{array}{cc}
0 & 1 \\
-\omega_{n}^{2} & -2 \zeta \omega_{n}
\end{array}\right]\left[\begin{array}{l}
x_{1} \\
x_{2}
\end{array}\right]+\left[\begin{array}{l}
0 \\
1
\end{array}\right] \Delta P_{L} \\
y & =\left[\begin{array}{ll}
1 & 0 \\
0 & 1
\end{array}\right]\left[\begin{array}{l}
x_{1} \\
x_{2}
\end{array}\right]
\end{aligned}
$$


The parameters $\omega_{n}$ and $\zeta$ are

$$
\begin{gathered}
\omega_{n}=\sqrt{\frac{\pi f_{o} P_{s}}{H}} \\
\zeta=\frac{D}{2} \sqrt{\frac{\pi f_{o}}{H P_{s}}}
\end{gathered}
$$

The meanings of the system variables and parameters are given in Table 1.

Table 1. System variables and parameters description

\begin{tabular}{cl}
\hline Variable & Description \\
\hline$x_{1}$ & Rotor angle in rad \\
$x_{2}$ & Frequency in $\frac{\mathrm{rad}}{\mathrm{sec}}$ \\
$\omega_{n}$ & Natural frequency in $\frac{\mathrm{rad}}{\mathrm{sec}}$ \\
$\zeta$ & Damping ratio \\
$D$ & Damping constant \\
$H$ & Per unit inertia constant \\
$f_{o}$ & Operating frequency in $\mathrm{Hz}$ \\
$\Delta P_{L}$ & Per unit input load deviation \\
$P_{s}$ & Synchronizing power coefficient \\
\hline
\end{tabular}

\subsection{The System T-S Fuzzy Model}

To set up the T-S fuzzy rule base, we first assume that the inputs to the rule premises are the states $\left(x_{1}, x_{2}\right)$. We select four operating points $-x_{0}=\left\{x_{01}, x_{02}, x_{03}, x_{04}\right\}$ to establish the required four local linear models in the output or consequence of the T-S fuzzy rules. If we assign two fuzzy linguistic values of positive small (PS) and positive $(\mathrm{P})$ to each input with notations

$$
\begin{aligned}
& x_{1}:\left\{M_{1}^{1}, M_{1}^{2}\right\} \\
& x_{2}:\left\{M_{2}^{1}, M_{2}^{2}\right\}
\end{aligned}
$$

we then formulate a total of four T-S fuzzy rules excluding the disturbance as follows.

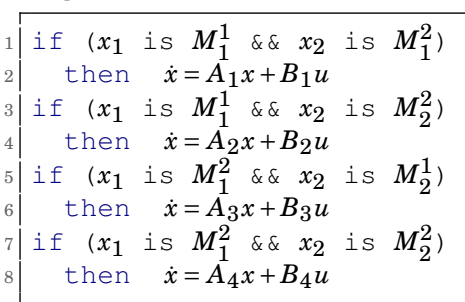

The overall T-S fuzzy model would take the form of (6).

\section{Simulation}

To set up the closed loop system for numerical evaluation, we state the following remarks.

1. The universe of discourse of the state is $X=[0.001,1]$.

2. Membership function is triangular expressed in (5).

In Table 2 are the states, centres of membership function shapes and operating points as in 2011, Shehu and Dan-Isa proposed ${ }^{[15]}$ here with slight modification in range and notations. The parameters specifications are given in Table 3.

Using the operating points we obtain the four distinct

\begin{tabular}{|c|c|c|}
\hline States & CTMF & $\mathrm{OP}$ \\
\hline$x_{1}=\left[\begin{array}{ll}\frac{1}{10} & 1\end{array}\right]$ & $\begin{array}{l}M_{1}^{1}=\left[\begin{array}{lll}0 & \frac{1}{8} & \frac{1}{4}\end{array}\right] \\
M_{1}^{2}=\left[\begin{array}{lll}\frac{1}{8} & \frac{1}{4} & \frac{1}{2}\end{array}\right]\end{array}$ & $\begin{array}{l}x_{1}^{1}=\frac{11}{100} \quad x_{1}^{2}=\frac{1}{4} \\
x_{1}^{3}=\frac{9}{20} \quad x_{1}^{4}=\frac{1}{2}\end{array}$ \\
\hline$x_{2}=\left[\begin{array}{ll}\frac{3}{1000} & \frac{9}{1000}\end{array}\right]$ & $\begin{aligned} M_{2}^{1} & =\left[\begin{array}{lll}0 & \frac{1}{400} & \frac{1}{200}\end{array}\right] \\
M_{2}^{2} & =\left[\begin{array}{lll}\frac{1}{500} & \frac{1}{200} & \frac{1}{100}\end{array}\right]\end{aligned}$ & $\begin{array}{ll}x_{2}^{1}=\frac{1}{1000} & x_{2}^{2}=\frac{1}{200} \\
x_{2}^{3}=\frac{7}{1000} & x_{2}^{4}=\frac{1}{100}\end{array}$ \\
\hline
\end{tabular}
state matrices $A=\left\{A_{i}\right\}$ in (22), (23), (24) and (25); and $B=\left\{B_{i}\right\}$ in (26) respectively.
Table 2. Fuzzy system set variables and parameters values

CTMF: Centres of Triangular Membership Functions

OP: Operating Points

Table 3. Power network parameter constants

\begin{tabular}{|l|l|l|}
\hline $\mid V=1$ p.u. & $X=\frac{13}{20}$ p.u. & $f_{o}=50 \mathrm{~Hz}$ \\
\hline$D=\frac{69}{500}$ & $H=8 \frac{89}{100} \frac{M J}{M V A}$ & $E_{o}=1 \frac{7}{20}$ p.u. \\
\hline$\delta_{o}=\frac{7 \pi}{75} \mathrm{rad} \mid \cos \theta=\frac{4}{5}$ & $P_{s}=1 \frac{99}{500}$ p.u. \\
\hline
\end{tabular}

$$
\begin{gathered}
A_{1}=\left[\begin{array}{cc}
0 & \frac{1}{1000} \\
-3 \frac{8647}{10000} & \frac{-3}{1250}
\end{array}\right] \\
A_{2}=\left[\begin{array}{cc}
0 & \frac{1}{200} \\
-8 \frac{3917}{5000} & \frac{-61}{5000}
\end{array}\right] \\
A_{3}=\left[\begin{array}{cc}
0 & \frac{7}{1000} \\
-15 \frac{8101}{10000} & \frac{-171}{10000}
\end{array}\right] \\
A_{4}=\left[\begin{array}{cc}
0 & 1 \\
-17 \frac{1417}{2500} & \frac{-61}{2500}
\end{array}\right] \\
B_{1}=B_{2}=B_{3}=B_{4}=\left[\begin{array}{l}
0 \\
1
\end{array}\right]
\end{gathered}
$$

\subsection{Simulation Results}

We may evaluate the system performance in cases as follows.

Case 1: Open-loop with nominal parameter values ( $D=0.138, P_{s}=1.988$ p.u. $)$.

Case 2: Open-loop with damping reduced $(D=0.0138$, $P_{s}=1.988$ p.u.).

Case 3: Open-loop with negative power coefficient $\left(D=0.0138, P_{s}=-1.988\right.$ p.u. $)$.

Case 4: Closed-loop with the designed controller under worst parameter condition $\left(D=0.0138, P_{s}=\right.$ -1.988 p.u.).

Applying 1 p.u. load demand, we obtain the system responses in Fig. 3.

\section{Discussion}

To understand the capability of the designed controller we first obtain results of the networks' frequency when in open loop condition at nominal and varied parameter conditions. At nominal parameter values, the network response of the rotor angle is shown in Fig. 3(a) and the load frequency in the steady state settles at the pre-set value of $50 \mathrm{~Hz}$ as shown in Fig. 3(b). 
(a)

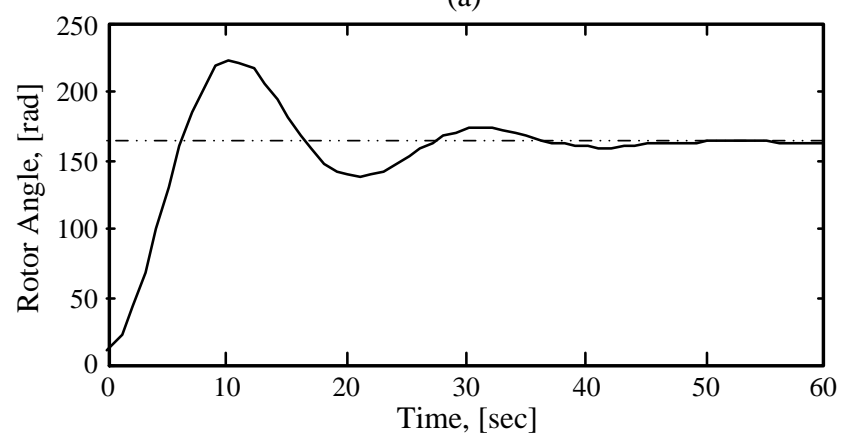

(c)

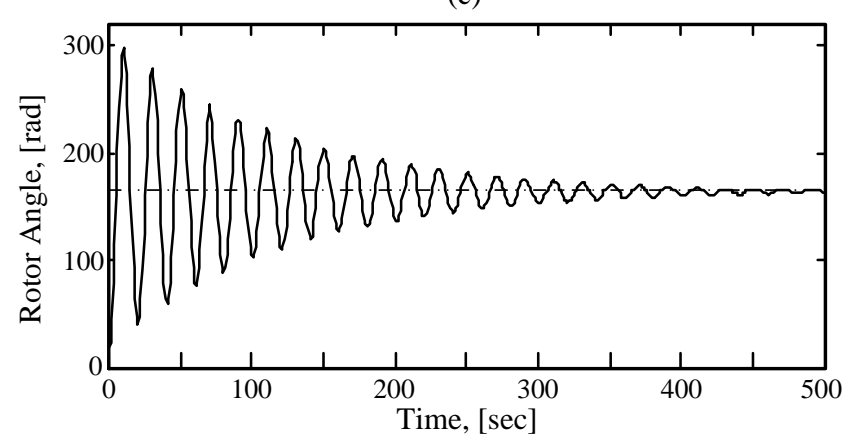

(e)

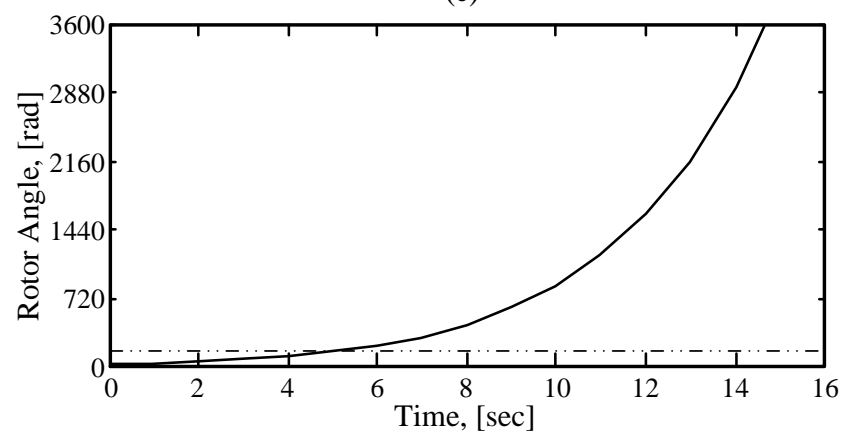

(g)

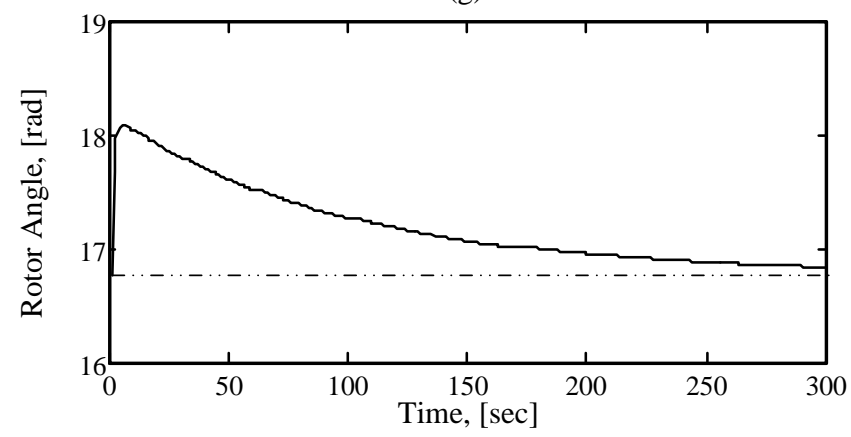

(b)

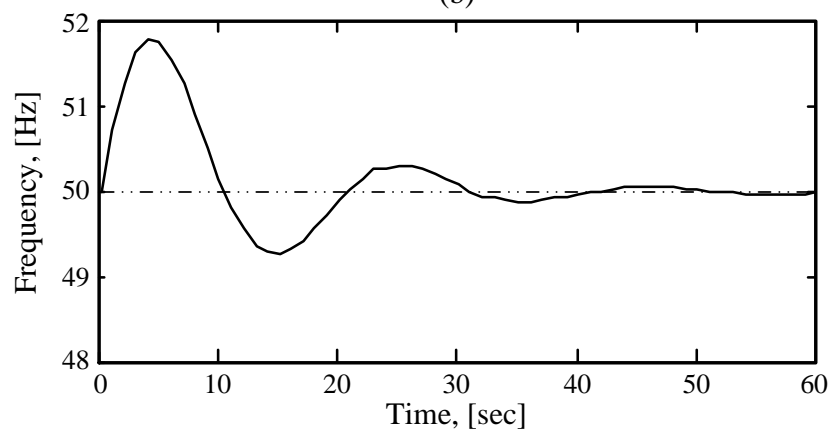

(d)

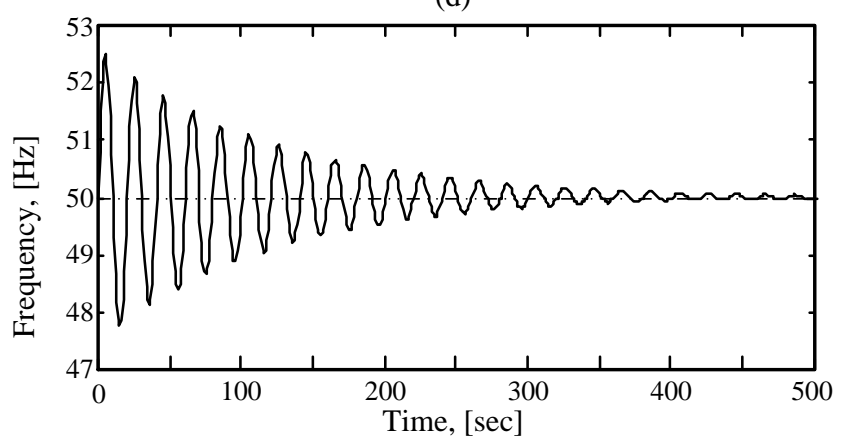

(f)

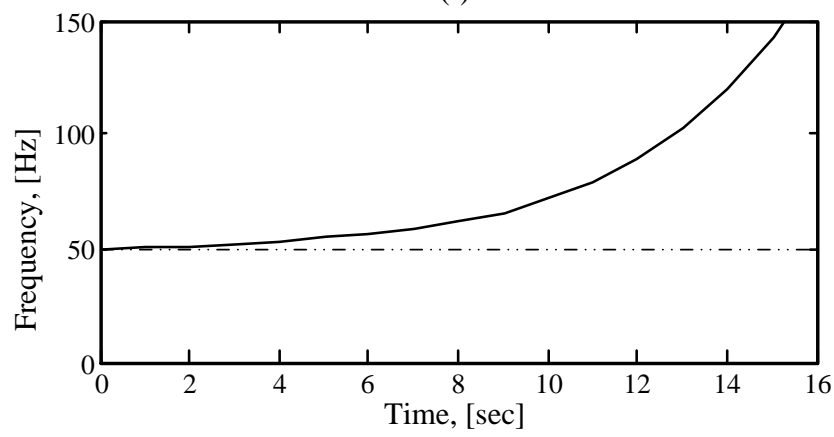

(h)

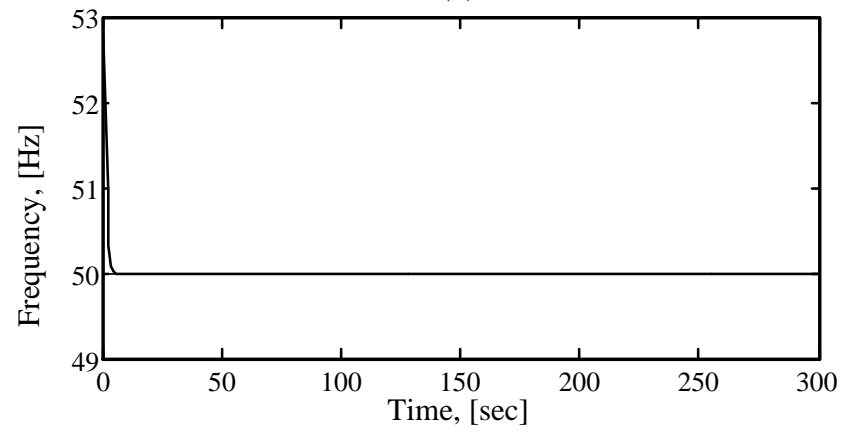

Fig. 3. Open-loop network response of the (a) rotor angle and (b) frequency at nominal $D$ and $P_{s}$; open-loop network response of the (c) rotor angle and (d) frequency at varying parameter values of $D=0.0138$ ( $D$ reduced to $10 \%$ ) and $P_{s}=1.988$ (nominal); open-loop network response of the (e) rotor angle and (f) frequency at varying parameter values of $D=0.138$ (nominal) and $P_{s}=-1.988$ (varied - worst case); and closed-loop system response of the (g) rotor angle and (h) frequency at worst parameter settings of $D=0.0138(D$ reduced to $10 \%$ ) and $P_{s}=-1.988$ (worst case). 
This performance was also observed when damping is reduced however, with severe oscillation during the transient phase of the rotor angle's response as shown in Fig. 3(c), and the response of the frequency is shown in Fig. 3(d). At negative power coefficient the rotor angle and frequency results are unbounded (unstable) as seen in Fig. 3(e) and Fig. 3(f).

Unlike in the case of PDC in the frame work of Linear Matrix Inequality result reported by Shehu and Dan-Isa ${ }^{[15]}$, here the fuzzy pole placement based controller even at the combine reduced damping and negative power coefficient parameter values it stabilizes the power network, providing good transient and excellent steady state performances (see Fig. 3(g) and Fig. 3(h)).

\section{Conclusion}

In the paper we presented a fuzzy pole placement based controller design. We applied the designed controller for the control of load frequency in a single machine and single area power generation network. First we represent the network with its equivalent T-S fuzzy model. Based on the fuzzy model, we formulate a PDC design determining the state feedback gain using a conventional pole placement technique. Simulation results obtained shows that the controller stabilizes the power network despite severe changes in the power networks' parameters values.

\section{REFERENCES}

[1] I. Elamvazuthi, P. Vasant, and J. F. Webb, "The application of mamdani fuzzy model for auto zoom function of a digital camera," arXiv preprint arXiv:1001.2279, 2010.

[2] H. Ying, "Structure and stability analysis of general mamdani fuzzy dynamic models," International journal of intelligent systems, vol. 20, no. 1, pp. 103-125, 2005.

[3] G. Castellano, A. M. Fanelli, and C. Mencar, "Design of transparent mamdani fuzzy inference systems.” in HIS. Citeseer, 2003, pp. 468-477.

[4] Z. Tečec, I. Petrović, and J. Matuško, "A takagi-sugeno fuzzy model of synchronous generator unit for power system stability application," Automatica: Journal for Control, Measurement, Electronics, Computing \& Communications, vol. 51, no. 2, pp. 127-137, 2010.

[5] F. Khaber, K. Zehar, and A. Hamzaoui, "State feedback controller design via takagi-sugeno fuzzy model: Lmi approach." International Journal of Computational Intelligence, vol. 2, no. 3, 2006.

[6] A. Abdelkrim, C. Ghorbel, and M. Benrejeb, "Lmi-based tracking control for takagi-sugeno fuzzy model." International Journal of Control \& Automation, vol. 3, no. 2, 2010.

[7] J. I. Corcau and E. Stoenescu, "Fuzzy logic controller as a power system stabilizer," International Journal of Circuits, Systems and Signal Processing, vol. 3, no. 1, pp. 266-273, 2007.

[8] J. Bondia, A. Sala, J. Picó, and M. A. Sainz, "Controller design under fuzzy pole-placement specifications: an interval arithmetic approach," Fuzzy Systems, IEEE Transactions on, vol. 14, no. 6, pp. 822-836, 2006.

[9] W. El Messoussi, O. Pagès, and A. El Hajjaji, "Robust pole placement for fuzzy models with parametric uncertainties: An lmi approach." in EUSFLAT Conf., 2005, pp. 810-815.

[10] S. K. Hong and Y. Nam, "Stable fuzzy control system design with pole-placement constraint: an lmi approach," Computers in Industry, vol. 51, no. 1, pp. 1-11, 2003.

[11] K. Tanaka and H. O. Wang, Fuzzy control systems design and analysis: a linear matrix inequality approach. John Wiley \& Sons, 2004.

[12] J. Li, D. Niemann, H. Wang, and K. Tanaka, "Parallel distributed compensation for takagi-sugeno fuzzy models: multiobjective controller design," in American Control Conference, 1999. Proceedings of the 1999, vol. 3. IEEE, 1999, pp. 1832-1836.

[13] R. C. Dorf, Modern control systems. Addison-Wesley Longman Publishing Co., Inc., 1995.

[14] J. J. Grainger and W. D. Stevenson, Power system analysis. McGraw-Hill New York, 1994, vol. 621.

[15] R. S. Shehu and A. Dan-Isa, "Simulation of a single power system network using linear matrix inequality based fuzzy feedback control," Nigerian Journal of Engineering, pp. 126-132, 2011. 